\title{
Parameter identification of Droop model: an experimental case study
}

\author{
Micaela Benavides ${ }^{1} \cdot$ Anne-Lise Hantson $^{1} \cdot$ Jan Van Impe $^{2} \cdot$ \\ Alain Vande Wouwer ${ }^{1}$
}

Received: 6 January 2015/Accepted: 20 May 2015/Published online: 2 June 2015

(c) Springer-Verlag Berlin Heidelberg 2015

\begin{abstract}
Mathematical modeling and the development of predictive dynamic models are of paramount importance for the optimization, state estimation, and control of bioprocesses. This study is dedicated to the identification of a simple model of microalgae growth under substrate limitation, i.e., Droop model, and describes the design and instrumentation of a lab-scale flat-plate photobioreactor, the associated on-line and off-line instrumentation, the collection of experimental data, and the parameter identification procedure. In particular, a dedicated methodology for parameter identification is discussed, including the determination of an initial parameter set using an analytical procedure, the selection of a cost function, the evaluation of confidence intervals as well as direct and cross-validation tests.
\end{abstract}

Keywords Mathematical modeling - Parameter estimation · Droop model · Photobioreactor · Bioprocesses

Micaela Benavides

Micaela.Benavides@umons.ac.be

Jan Van Impe

Jan.VanImpe@cit.kuleuven.be

Alain Vande Wouwer

Alain.VandeWouwer@umons.ac.be

1 BioSys, University of Mons, Boulevard Dolez 31, 7000 Mons, Belgium

2 Department of Chemical Engineering, Catholic University of Leuven, 3001 Leuven, Belgium

\section{Introduction}

Research and applications of microalgal cultivation have experienced a fantastic boom in the last two decades due to a renewed interest in alternative energy sources, and the potential of microalgae to produce large quantities of neutral lipids (e.g., 20-50 \% dry cell weight) for biodiesel production [1, 2]. Besides, microalgae have a large spectrum of applications ranging from the production of pigments, cosmetics, animal fodder to wastewater treatment $[2,3]$.

These ever increasing applications motivate the development of process monitoring and control, aiming at improving process reliability and productivity. In this context, it is necessary to develop predictive dynamic models that could be used for the design of software sensors [4], i.e., state estimation techniques blending the information of the process model with available instrumentation to reconstruct nonmeasured variables, or for the design of optimizing controllers.

Several models describing microalgal growth in open pounds, or bioreactors, have been proposed, for instance in [5-7]. A good overview of recent developments is given in [8]. One of the earlier, and nowadays classical, models of microalgal cultivation under substrate limitation has been originally developed by Droop [9]. This model uncouples substrate uptake (leading to the formation of a so-called internal quota), and biomass growth. It is often the corner stone of more elaborate models, including additional effects such as light photoacclimation and inhibition [8, 10].

Even though Droop model is well established and there are many reports of successful applications, there is still a need for the development of a systematic identification procedure and its experimental evaluation. The objective of this work is to assess the experimental effort needed to 
estimate the parameters of Droop model, and to discuss an experimental case study, based on a lab-scale flat-plate photobioreactor. Attention is focused on the on-line and off-line instrumentation, and the development of an identification procedure including initialization, cost function selection, evaluation of confidence intervals, and model validation.

This paper is organized as follows. The next section introduces Droop model, while Sect. 3 presents the laboratory set-up including the flat-panel photobioreactors and their associated off-line and on-line instrumentation. Section 4 is dedicated to the parameter estimation procedure and discusses data collection, model identification, and validation. Finally, in Sect. 5, some conclusions are drawn and prospects are envisioned.

\section{Droop model}

Droop model [9] describes the growth of microalgae cultivated in a photobioreactor, under constant temperature and illumination conditions. Droop model uncouples growth from substrate uptake, leading to the definition of an internal cell quota (i.e., the internal nutrient quantity per unit of biomass), and describes the growth rate as a function of this internal quota only. Below a so-called subsistence quota, the algae cannot grow.

Originally, Droop considered vitamin $\mathrm{B}_{12}$ as a limiting nutrient. Since then, it has been demonstrated that the model is applicable to other limiting substrates such as nitrate, phosphate, and silicate [5]. In our work, nitrogen is the limiting nutrient as considered in [8]. Nitrogen depletion enhances the accumulation of lipids within the microalgae, and corresponds to bioreactor operating conditions used for the production of biofuels.

The mass balance differential equation model presented in Eq. (1) involves three state variables: the concentration of biomass $X$, the concentration of substrate $S$, and the intracellular quota $Q$.

$$
\begin{aligned}
\dot{X} & =\mu(Q) X-D X \\
\dot{S} & =-\rho(S) X+D\left(S_{\mathrm{in}}-S\right) \\
\dot{Q} & =\rho(S)-\mu(Q) Q
\end{aligned}
$$

The dilution rate $D=F_{\text {in }} / V$ is the ratio of the inlet flowrate to the volume of the culture.

The uptake rate $\rho(S)$ is defined by a Michaelis-Menten law:

$\rho(S)=\rho_{\mathrm{m}} \frac{S}{S+K_{\mathrm{s}}}$,

where $K_{\mathrm{s}}$ is the half saturation constant and $\rho_{\mathrm{m}}$ is the maximum inorganic nitrogen absorption rate.
Table 1 Model variables and parameters

\begin{tabular}{ll}
\hline Variables & Parameters \\
\hline Biomass, $X(\mathrm{mgC} / \mathrm{L})$ & $\begin{array}{c}\text { Maximum absorption rate, } \\
\rho_{\mathrm{m}}(\mathrm{mgN} / \mathrm{mgC} / \text { day })\end{array}$ \\
Substrate, $S(\mathrm{mgN} / \mathrm{L})$ & Half saturation constant, $K_{\mathrm{s}}(\mathrm{mgN} / \mathrm{L})$ \\
Intracellular quota, $Q(\mathrm{gN} / \mathrm{gC})$ & Maximum growth rate, $\mu_{\mathrm{m}}($ per day) \\
& Minimal cell quota, $Q_{0}(\mathrm{mgN} / \mathrm{mgC})$ \\
& Inflow substrate, $S_{\text {in }}(\mathrm{mgN} / \mathrm{L})$ \\
& Dilution rate, $D($ per day $)$ \\
\hline
\end{tabular}

The specific growth rate is defined as a function of the intracellular quota $(Q)$ as follows:

$\mu(Q)=\mu_{\mathrm{m}}\left(1-\frac{Q_{0}}{Q}\right)$

where $Q_{0}$ is the minimal cell quota or subsistence quota, i.e., the parameter identified by Droop under which the algae do no longer grow, and $\mu_{\mathrm{m}}$ is the maximum specific growth rate. In [11], it has been proved that the cell quota remains between two bounds: $Q_{0} \leq Q \leq Q_{\mathrm{m}}$, where the maximum cell quota $Q_{\mathrm{m}}$ obtained in conditions of nonlimiting nutrient is given by

$$
Q_{\mathrm{m}}=Q_{0}+\frac{\rho_{\mathrm{m}}}{\mu_{\mathrm{m}}}
$$

The physical units of the several variables and parameters considered in this work are presented in Table 1.

In this work, the saline microalgae Dunaliella tertiolecta is cultivated in a culture medium with limiting quantities of nitrate. The experimental set-up is described in the next section. The cultures are achieved in batch mode $(D=0)$ so that the model reduces to

$$
\begin{aligned}
\dot{X} & =\mu(Q) X \\
\dot{S} & =-\rho(S) X \\
\dot{Q} & =\rho(S)-\mu(Q) Q .
\end{aligned}
$$

\section{Materials and methods}

\section{Photobioreactor set-up}

The cultivation of microalgae is achieved in an in-house lab-scale process, which consists of a flat-panel reactor, a light source, and a set of probes for on-line measurements. Figure 1a shows a sketch of the photobioreactor (PBR), which has a culture volume of $13 \mathrm{~L}$ and is illuminated from one side by a set of six fluorescent tubes placed vertically and parallel to the front side of the reactor, with the same height and width as the reactor. These fluorescent tubes of 
Fig. 1 a Schematic representation of the PBR, b actual view of the PBR

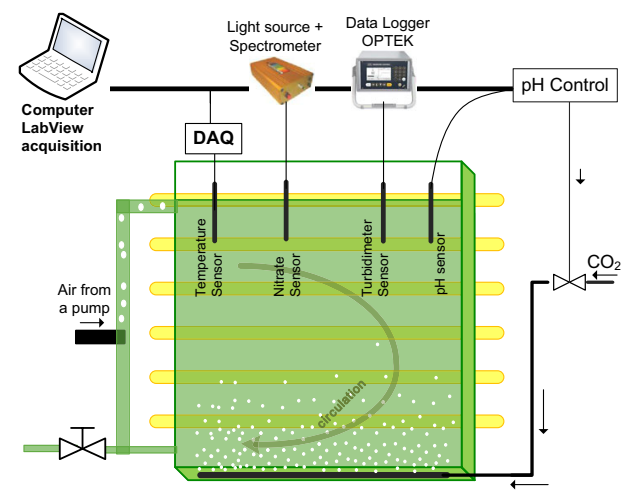

(a)

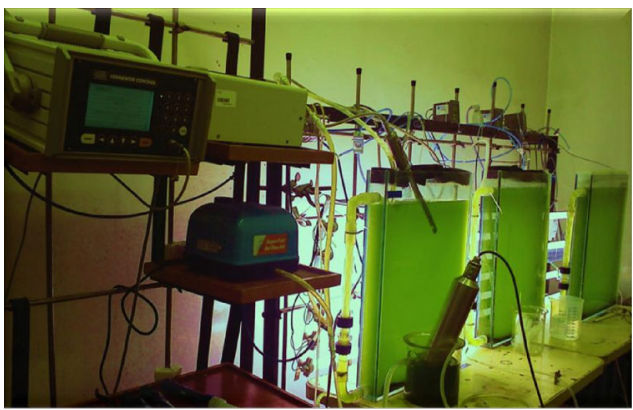

(b)
$18 \mathrm{~W}$ each are dimmable and used generally in horticulture applications (Fluora $18 \mathrm{~W} / 77$, Osram). The main emitted wavelengths are located in the visible spectrum [blue $(430 \mathrm{~nm})$ and red $(620 \mathrm{~nm})]$ to promote photosynthesis. A constant light intensity of $150 \mu \mathrm{mol} / \mathrm{m}^{2} / \mathrm{s}$ was measured.

To have a well-distributed culture medium, the air-lift principle is used, with an air pump delivering compressed air at $0.1 \mathrm{~L} / \mathrm{min}$.

Pure gaseous carbon dioxide $\mathrm{CO}_{2}$ is injected at the bottom of the reactor using a microporous diffuser. The $\mathrm{CO}_{2}$ flow is modulated by an electromagnetic valve, controlled by a $\mathrm{pH}$ control module. Figure $1 \mathrm{~b}$ shows a picture of the laboratory set-up.

\section{Microalgal culture}

Batch experiments were performed for the culture of the microalgal $D$. tertiolecta. The medium consists of the following components: $\mathrm{NaCl}(10 \mathrm{~g} / \mathrm{L}), \mathrm{MnCl}_{2} \cdot 6 \mathrm{H}_{2} \mathrm{O}(1.5 \mathrm{~g} / \mathrm{L})$, $\mathrm{KNO}_{3}(0.2446 \mathrm{~g} / \mathrm{L}), \mathrm{MgSO}_{4} \cdot 7 \mathrm{H}_{2} \mathrm{O}(0.5 \mathrm{~g} / \mathrm{L}), \mathrm{CaCl}_{2} \cdot 2 \mathrm{H}_{2} \mathrm{O}$ $(0.2 \mathrm{~g} / \mathrm{L}), \mathrm{KCl}(0.2 \mathrm{~g} / \mathrm{L}), \mathrm{K}_{2} \mathrm{HPO}_{4}(0.045 \mathrm{~g} / \mathrm{L})$, and $\mathrm{mi}-$ cronutrients. The medium was prepared using deionized water.

\section{Data collection}

An advantage of the flat-panel PBR design is that sensors can be installed through the top of the reactor, according to the project needs. For monitoring the microalgae growth, several variables are measured on-line, including $\mathrm{pH}$, temperature, biomass, and nitrate concentrations. The online probes are connected to a portable PC to collect the measurements using LabView data acquisition tools. Other variables are measured off-line, including the intracellular nitrogen quota, as well as additional measurements of biomass and nitrate concentrations (which can be used as a cross-check).
The $\mathrm{pH}$ probe is an electrode, which is connected to an on/off pH controller unit (HANNA Instruments BL 931700) with a programmable set point. In this work, the $\mathrm{pH}$ is established at 7.5 and an error of \pm 0.2 is observed.

Temperature is measured using a probe EI-1034 together with a DAQ module LABJACK U3. The temperature probe consists of a silicon-type temperature sensor mounted in a waterproof stainless steel tube. It uses the LM34 sensor from the National Semiconductor, with a typical room temperature accuracy of $\pm 0.4^{\circ} \mathrm{F}$, and a linear voltage output in relation to the temperature. The cultures were performed in a laboratory environment where the temperature is kept above $23{ }^{\circ} \mathrm{C}$ and with a maximum temperature of $28{ }^{\circ} \mathrm{C}$.

Different methods are applied to measure the biomass concentration, nitrate concentration, and the internal nitrogen. The following subsections explain these methods.

\section{Biomass concentration acquisition methods}

There are a number of off-line and on-line methods, which have been developed to sense and quantify biomass. In this work, two optical density methods were applied to measure the biomass. The first one is an off-line measurement method performed with a Shimadzu UV-mini 1240 spectrophotometer. The second one is an on-line method, which uses an optical Optek sensor model ASD19-N to measure the optical density in concentration units. In both cases, the dry weight method was used as reference to correlate the absorbance measurements with the biomass concentration.

Spectrophotometer. This is an optical method where the value of the biomass concentration is measured indirectly by light absorption. For each measurement, a sample ( $4 \mathrm{~mL}$ of medium) is taken from the photobioreactor, put into a square cell, and measured with a spectrophotometer Shimadzu UV-mini 1240, Fig. 2a.

Since the maximum absorbance value for the target microalgae was observed at $680 \mathrm{~nm}$, this wavelength was selected to measure the absorbance of the sample. 
Fig. 2 a Optek sensor (http:// www.optek.com). b Calibration curve for the absorbance measurement in cultures of $D$. tertiolecta

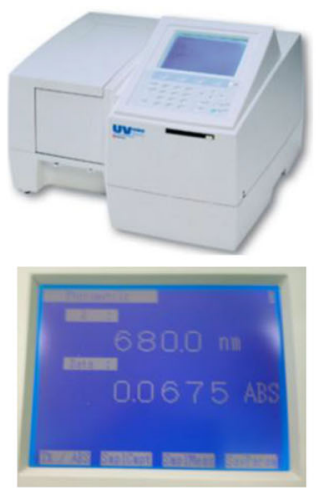

(a)

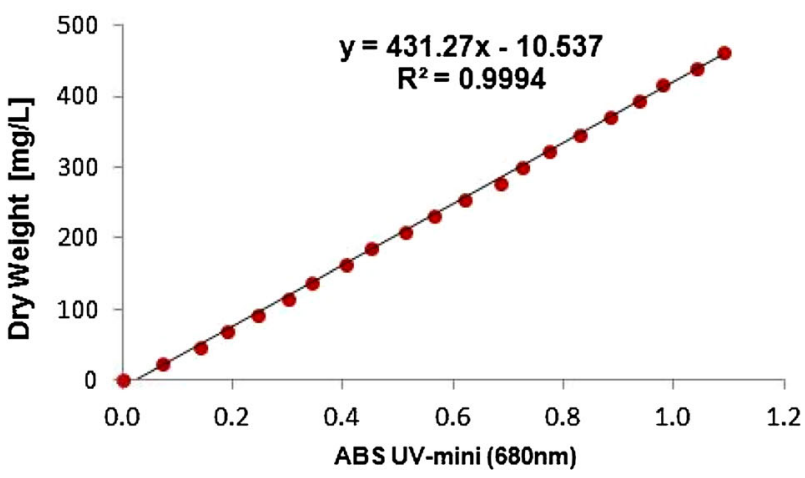

(b)
Fig. 3 a Optek sensor (http:// www.optek.com). b Correlation between $\mathrm{CU}$ (Optek) and dry biomass of the strain $D$. tertiolecta

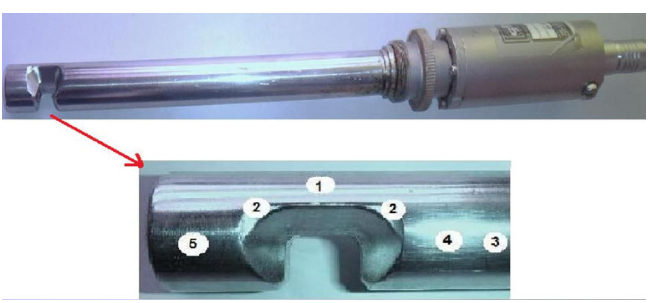

(a)

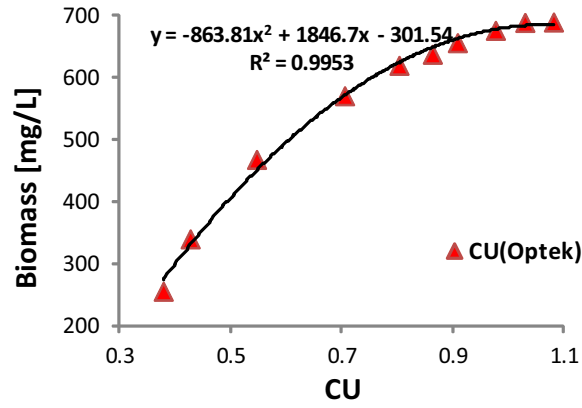

(b)
In order to obtain the biomass concentration measurement in $\mathrm{mg} / \mathrm{L}$, it is necessary to establish a correlation between the optical density and the dry weight. Figure $2 b$ shows this linear correlation (optical density at $680 \mathrm{~nm}$ and dry weight of a sample diluted 20 times), with a coefficient of correlation of 0.9994 . The model equation is given by

Dry biomass $(\mathrm{mg} / \mathrm{L})=431.27 \times \mathrm{OD}_{680}-10.537$

The sensitivity of this calibration is $0.002 \mathrm{Abs} / \mathrm{mg} / \mathrm{L}$. The photometric accuracy of the equipment is $\pm 0.005 \mathrm{Abs}$ (at $1.0 \mathrm{Abs}$ ) and $\pm 0.003 \mathrm{Abs}$ (at $0.5 \mathrm{Abs}$ ) and the measurement range is from -0.3 to 3.0 Abs. Since the calibration curve is established with a maximum value of $1.1 \mathrm{Abs}$, the maximum measurable concentration value is $464 \mathrm{mg} / \mathrm{L}$. To measure concentration values larger than this value, it is necessary to dilute the sample.

On-line turbidity (Optek probe). Another method used to measure the biomass concentration is based on the near infrared (NIR) absorption probe (Optek, model ASD19-N), as shown in Fig. 3a. The main advantage of the Optek probe is that it can take the measurements on-line, been immersed into the photobioreactor.

Results from the Optek equipment are displayed in Concentration Units (CU-the measurement range is from 0 to $4 \mathrm{CU}$ ).
Following the same procedure as in the previous section, a calibration curve of the Optek sensor has been obtained. Moreover, ten samples were taken during the exponential growth of the microalgae $D$. tertiolecta and the biomass concentration indirectly obtained from the UV-mini was used as a reference. Figure $3 \mathrm{~b}$ shows the quadratic correlation between $\mathrm{CU}$ and biomass concentration with correlation coefficient of $R^{2}=0.9953$.

\section{Nitrate concentration acquisition methods}

The nitrate concentration in the medium is measured using absorption spectroscopy. This method uses the absorption properties of nitrate: when dissolved in water, nitrate absorbs the ultraviolet (UV) light at wavelengths of less than $250 \mathrm{~nm}$ [12]. Due to this self-absorption property, the nitrate concentration can be measured with the spectrophotometer Shimadzu UV-mini using the deuterium light source. Since the turbidity of the sample causes interference in the measurement of the absorbance, the nitrate concentration measurement takes two wavelength into account: 220 and $275 \mathrm{~nm}$. At $220 \mathrm{~nm}$, the measurement $\left(\mathrm{ABS}_{220}\right)$ considers the nitrate absorption plus the interference, while at $275 \mathrm{~nm}$, only the interference due to the turbidity $\left(\mathrm{ABS}_{275}\right)$ is considered. Consequently, the nitrate 
Fig. 4 a Absorbance spectrum of six dilutions of a nitrate solution. b Calibration curve

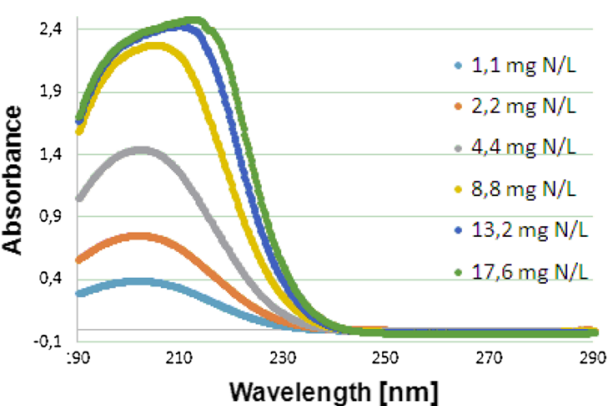

(a)

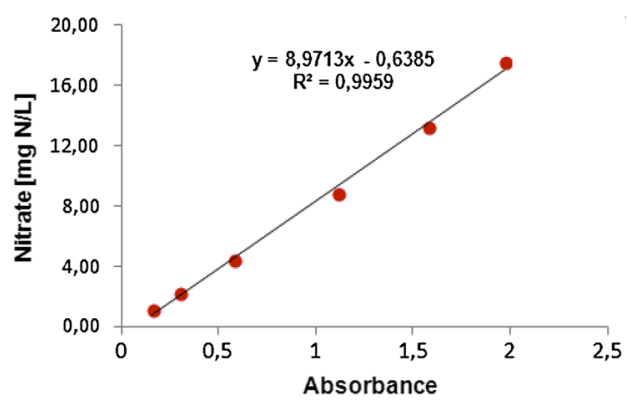

(b) concentration is obtained from the difference of both measurements $\mathrm{ABS}_{220}-\mathrm{ABS}_{275}$.

The calibration follows the manual method [13]. A relationship between absorbance and nitrate concentration is obtained by preparing six dilutions of a nitrate solution, from 1.1 to $17.6 \mathrm{mgN} / \mathrm{L}$. Figure 4a shows the spectrum of these sample dilutions using a wavelength from 190 to $290 \mathrm{~nm}$, performed with the Shimadzu UV-mini using a $5 \mathrm{~mm}$ quartz cell. For these results, demineralized water was used to set the zero absorbance. The absorbance difference $\left(\mathrm{ABS}_{220}-\mathrm{ABS}_{275}\right)$ is related with the nitrate concentration value to obtain the calibration curve, as shown in Fig. 4b.

Furthermore, it is possible to use this principle to obtain on-line measurements of nitrate concentration. To this end, an optical fiber immersed directly in the medium was used. A deuterium light source provides the light that will travel through the fiber optics into the medium and be detected by a spectrometer. The calibration procedure is the same as explained above.

\section{Intracellular nitrogen acquisition method}

To measure intracellular components, it is necessary to stop the metabolism very abruptly and to disintegrate the cells quantitatively. For this analysis, a Shimadzu TOC$\mathrm{V}_{\mathrm{CPH}}$ analyzer working with a Total Nitrogen Module (TNM-1, Shimadzu) is exploited. This unit works with chemiluminescence to measure total nitrogen. In this process, the sample is combusted to nitrogen monoxide and nitrogen dioxide. The nitrogen species reacts with ozone to form an excited state of nitrogen dioxide. Upon returning to the ground state, light energy is emitted. Then, total nitrogen (TN) is measured using a chemiluminescence detector.

The measurements can be taken over a wide range from 0.1 to $4000 \mathrm{mg} / \mathrm{L}$, and their accuracy has a coefficient of variation of $3 \%$ max. Shimadzu TNM-1 can also simultaneously measure the total organic carbon (TOC).
To proceed with the measure of total nitrate, the TNM-1 was calibrated using $100 \mathrm{mg} / \mathrm{L}$ of $\mathrm{KNO}_{3}$ standard solution, and the dilutions were obtained automatically from the unit. After calibration, the measurement is realized with a sample volume of $15 \mathrm{~mL}$ of microalgae which is centrifuged and washed two times with milli-Q water. The instrument achieves three replicated injections for each sample in order to have a reproducibility test and give the average value as a result.

\section{Parameter estimation}

\section{Background methodology}

The goal of parameter identification is to find the best or optimal value of the parameters that allows an accurate prediction of the experimental data by the model $\mathbf{M}(\theta)$. The identification problem is solved considering the output measurements, the control inputs, and the model structure. The method to select the optimal parameter value can be seen as the minimization of a cost function, or the prediction error denoted by $J$.

This latter function can be selected in various ways. The weighted least-squares method is the most popular choice, where the minimization of the following cost function with respect to the parameters $\theta$ leads to a nonlinear programming problem

$$
J(\theta)=\sum_{i=1}^{N}\left[\left(y_{i}(\theta)-y_{i, \text { meas }}\right)^{\mathrm{T}} \cdot W_{i}^{-1} \cdot\left(y_{i}(\theta)-y_{i, \text { meas }}\right)\right]
$$

In this expression, $y_{i \text {,meas }}$ is the measurement vector, $y_{i}$ is the prediction vector according to the model, $\theta$ is the parameter vector, $N$ is the number of measurement points, and $W_{i}^{-1}$ is the error-weighting matrix.

If the statistics of the measurement noise $v_{i}$ is considered, i.e., $y_{i \text {,meas }}=y_{i}+v_{i}$, then an optimal (in the 
maximum likelihood sense) choice for $W_{i}$ is the measurement error variance-covariance matrix $\Sigma_{i}$ at each time point.

The minimization of the cost function

$\hat{\theta}=\arg \min _{\theta} J(\theta)$

can be achieved with well-established software library tools. In this study, a Nelder-Mead algorithm, as implemented in the Matlab ${ }^{\mathrm{TM}}$ routine 'fminsearch' was used.

In order to evaluate the parameter estimate precision, the Fisher Information Matrix [14] can be evaluated, which is defined as follows:

$F=\sum_{i=1}^{N}\left(\frac{\partial y}{\partial \theta}\left(t_{i}\right)\right)^{\mathrm{T}} \Sigma_{i}^{-1}\left(\frac{\partial y}{\partial \theta}\left(t_{i}\right)\right)$

The FIM contains information about the measurement noise (via the inverse of the covariance matrix $\Sigma_{i}^{-1}$ ), and the output sensitivity function $\partial y / \partial \theta$ at each point $i$ in time. The estimation of the parameter variance-covariance matrix can be approximated from the inverse of the Fisher information matrix, as follows:

$C_{N}=F^{-1}$

Finally, the standard deviation of the estimated parameters $\hat{\theta}_{i}$ can be obtained from the square root of the $i$ th diagonal element of the parameter variance-covariance matrix:

$\sigma\left(\hat{\theta}_{i}\right)=\sqrt{C_{N}(i, i)}$.

With the standard deviation, it is possible to estimate the confidence intervals at a given confidence level:

$\left[\hat{\theta}_{i}-t_{\alpha,(N-p)} \sigma_{i} ; \hat{\theta}_{i}+t_{\alpha,(N-p)} \sigma_{i}\right]$,

where $\alpha$ is the desired significance level and the confidence level is $100(1-\alpha) \%, N$ is the number of measurement points and $p$ is the number of parameters in the model.

The coefficient of variation $(\mathrm{CV})$ can also be used, which is defined by

$\operatorname{CV}\left(\hat{\theta}_{i}\right)=\frac{\sigma\left(\hat{\theta}_{i}\right)}{\hat{\theta}_{i}} \cdot 100 \%$

\section{Initialization procedure}

As parameter estimation involves a nonlinear optimization problem which can be subject to local minima, it is important to start the optimization from a reasonable initial guess. A first estimate of the parameters can be obtained using an analytic procedure inspired from [7, 15]:

- From the biomass measurement, the initial exponential phase is used to estimate the maximum growth rate $\mu_{\max }$. In this phase, the mass balance equation (4) can be approximated as follows:

$$
\begin{aligned}
& \frac{\mathrm{dX}}{\mathrm{d} t}=\mu_{\max } X \\
& \ln \left(\frac{X}{X_{0}}\right)=\mu_{\max } t
\end{aligned}
$$

The parameter $\mu_{\max }$ is the slope of the straight line $\ln (X / X(0))$ vs time.

- The maximal and minimal intracellular nitrogen quotas, $Q_{\mathrm{m}}$ and $Q_{0}$, can be estimated from the time evolution of the internal quota measurements. $Q_{\mathrm{m}}$ is obtained at the time instant the substrate gets exhausted, while $Q_{0}$ is assessed when biomass growth stops.

- When the quota is at its maximum value, the maximum growth rate value $\mu_{\max }$ is observed, so that

$\mu_{\max }=\mu\left(Q_{\mathrm{m}}\right)=\mu_{\mathrm{m}}\left(1-\frac{Q_{0}}{Q_{\mathrm{m}}}\right)$

Solving this equation for the maximum growth rate $\mu_{\mathrm{m}}=\mu_{\max } Q_{\mathrm{m}} /\left(Q_{\mathrm{m}}-Q_{0}\right)$ gives an additional parameter estimate.

- At the beginning of the batch (large initial substrate concentration), $\rho(S) \approx \rho_{\mathrm{m}}$ and

$\frac{\mathrm{d} Q}{\mathrm{~d} t} \approx \rho_{\mathrm{m}}$

so that an estimate of the maximum absorption rate can be obtained from the initial slope of the graph of $Q(t)$.

- The half saturation constant corresponds to the substrate concentration $\left(S=K_{\mathrm{s}}\right)$ at which the absorption rate is half the maximum value $\left(\rho(S)=\rho_{\mathrm{m}} / 2\right)$. Considering that the absorption rate reaches this latter value when the substrate is almost consumed, an initial guess for the half saturation constant is obtained from the lower values of the substrate.

The parameter values shown in Table 2 are obtained from this initialization procedure. They will be used as the "initial guess" in the parameter identification algorithm.

\section{Nonlinear parameter estimation}

Parameter estimation is achieved so as to obtain more precise values for the parameters $\rho_{\mathrm{m}}, K_{\mathrm{s}}, \mu_{\mathrm{m}}, Q_{0}$. Besides,

Table 2 Initial parameter estimates

\begin{tabular}{llll}
\hline & Initial guess & First estimates & CV (\%) \\
\hline$\rho_{\mathrm{m}}$ & 0.048 & 0.0901 & 0.95 \\
$K_{\mathrm{s}}$ & 2.000 & 1.6499 & 4.96 \\
$\mu_{\mathrm{m}}$ & 1.137 & 1.9054 & 0.39 \\
$Q_{0}$ & 0.030 & 0.0350 & 0.12 \\
\hline
\end{tabular}


the initial conditions of the culture $X(0), S(0)$, and $Q(0)$, which are measured and are therefore subject to errors, can also be estimated so as to improve the model fit.

This task is performed by minimizing the cost function presented in Eq. 6, with a weighting matrix assuming no prior knowledge on the statistics of the data, but just scaling the data

$$
W=\operatorname{diag}\left[\max \left(X^{2}\right) \quad \max \left(S^{2}\right) \quad \max \left(Q^{2}\right)\right]
$$

After minimization of the cost function, an estimate of the standard deviation is obtained by

$$
\hat{\sigma}^{2}=\frac{J(\hat{\theta})}{N-p}
$$

The covariance matrix of the measurement errors can therefore be roughly estimated as $\Sigma \approx \hat{\sigma}^{2} W$.

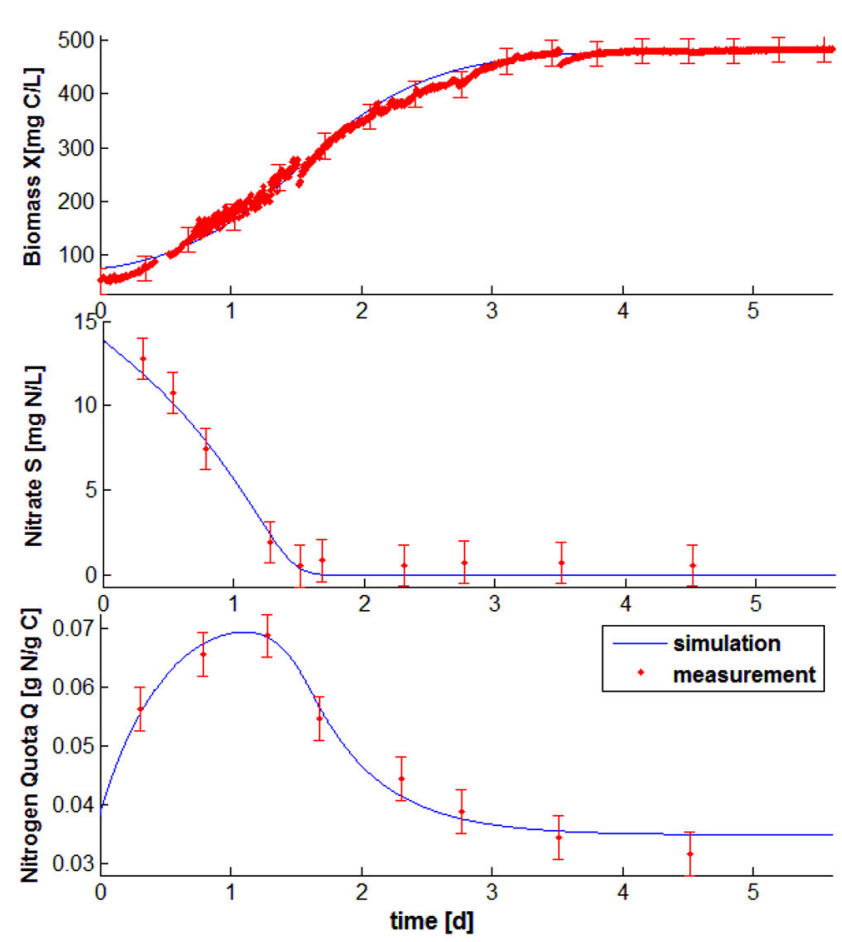

(a)
In a first attempt, the experimental data collected from two batch cultures (denoted in the following as first experiment and second experiment) are considered. Each culture has a duration of about five days, but differs in their initial conditions. The biomass concentration is measured continuously every 5 minutes, while the other measurements are obtained off-line.

Parameter estimation is achieved using the data of the first experiment only. The estimated parameters are listed in Table 2 (they are called first estimates). Figure 5a shows direct validation results, i.e., compares the model prediction, when using the estimated parameters, to the experimental data used in the evaluation of the cost function. The experimental data are represented by red dots (and $95 \%$ confidence intervals), while the model prediction is depicted by blue lines. The second experiment is used for cross-validation purposes, i.e., the identified model is
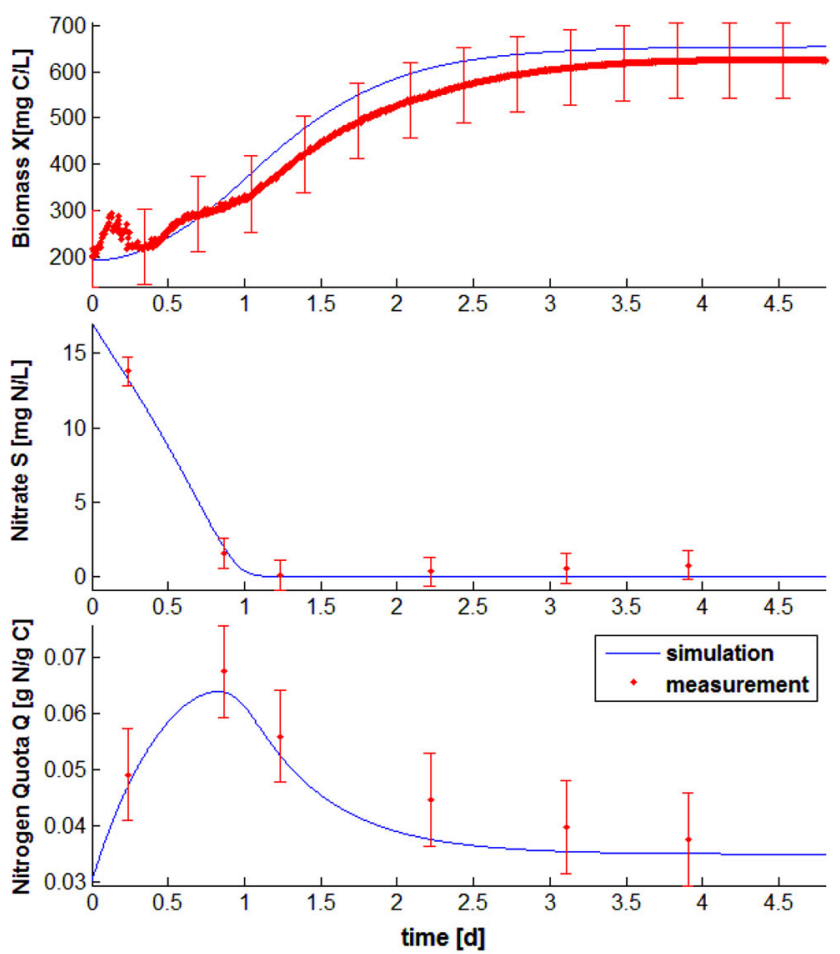

(b)

Fig. 5 Red dots experimental data, and blue lines model prediction. a Direct validation, b cross validation (color figure online)

Table 3 Parameter and initial condition estimates

\begin{tabular}{lllllclccc}
\hline & Initial guess & Second estimates & $\mathrm{CV}(\%)$ & \multicolumn{2}{l}{ Initial conditions } \\
\cline { 5 - 9 } & & & & & First exp. & $\mathrm{CV}$ & Second exp. & $\mathrm{CV}$ \\
\hline$\rho_{\mathrm{m}}$ & 0.048 & 0.0916 & 1.30 & $X(0)$ & 91.483 & 0.65 & 192.332 & 0.45 \\
$K_{\mathrm{s}}$ & 2.000 & 1.7499 & 6.38 & $S(0)$ & 14.5148 & 0.26 & 17.03 & 0.27 \\
$\mu_{\mathrm{m}}$ & 1.137 & 1.8102 & 0.55 & $Q(0)$ & 0.0347 & 0.95 & 0.0303 & 0.92 \\
$Q_{0}$ & 0.030 & 0.0369 & 0.15 & & & & & \\
\hline
\end{tabular}


challenged with fresh data (data that have not been used in the identification procedure), as shown in Fig. 5b. We can notice that the identified model does not fit very well in cross validation.

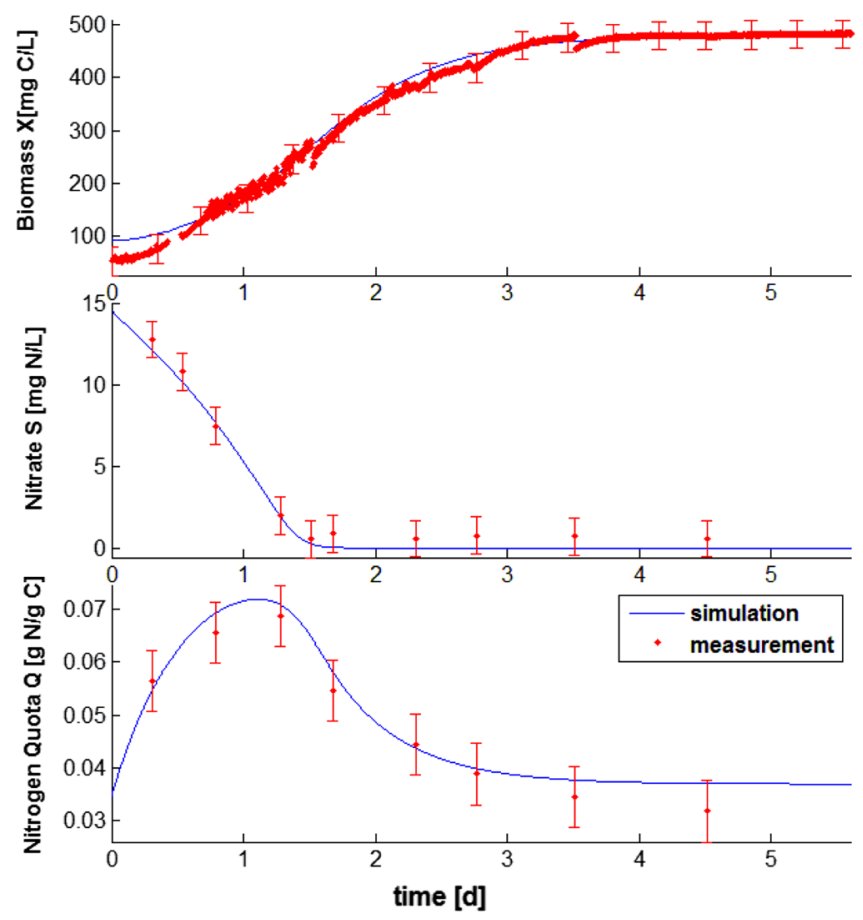

(a)
The number of experiments (in our case batch cultures) that have to be considered for parameter estimation depends on the number of parameters to estimate (in our case study, there are four kinetic parameters and three initial

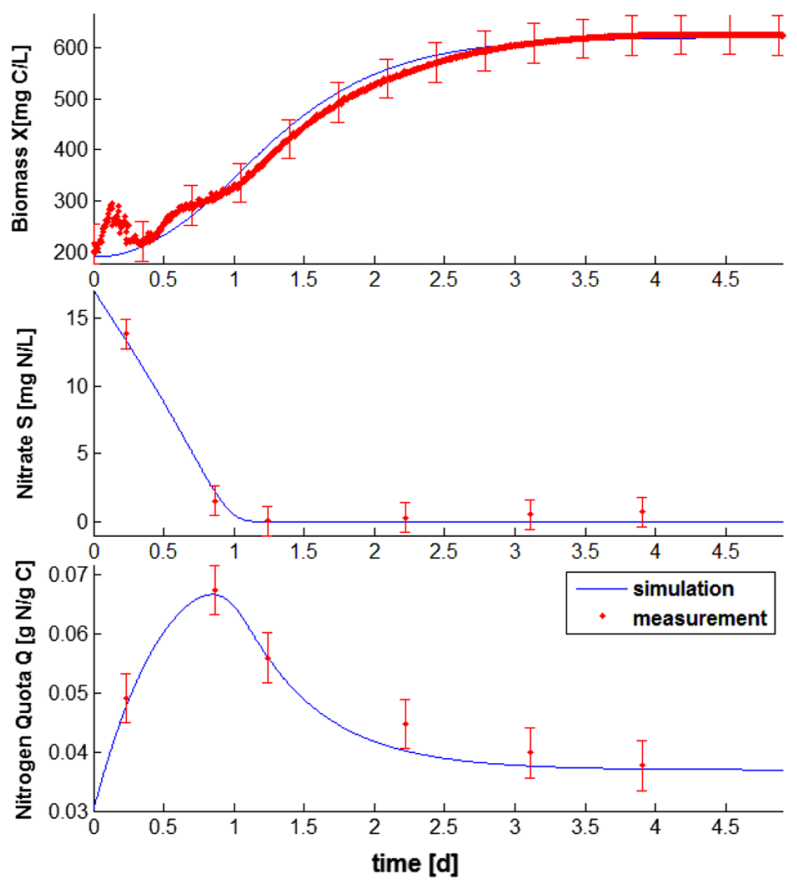

(b)

Fig. 6 Red dots experimental data, and blue lines model prediction-direct validation (color figure online)

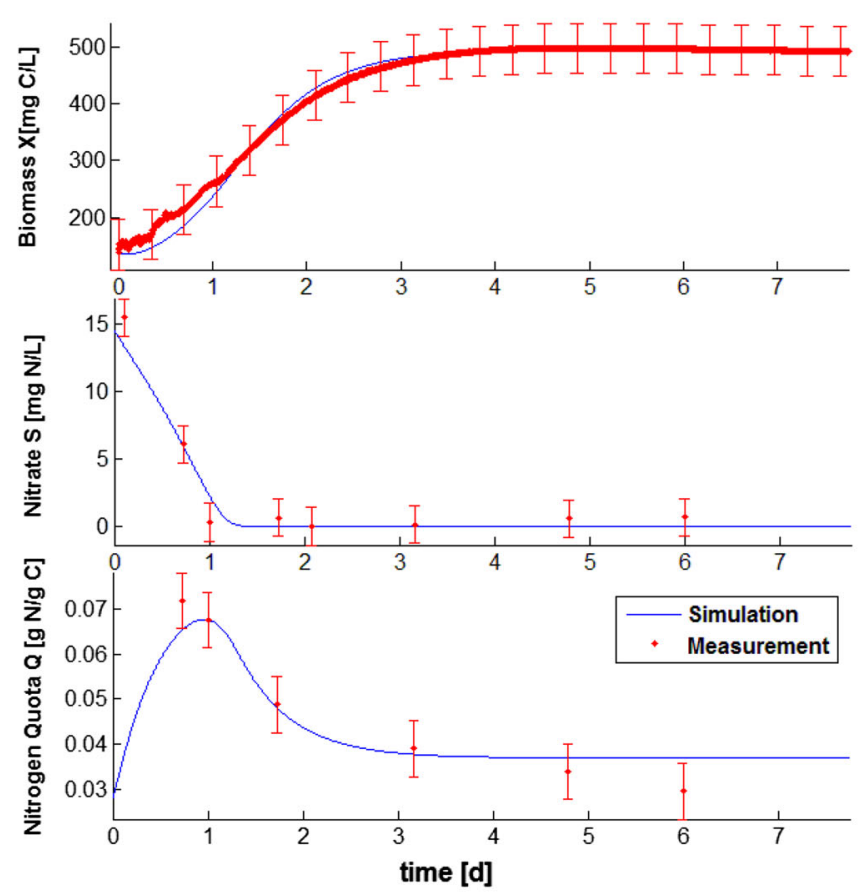

(a)

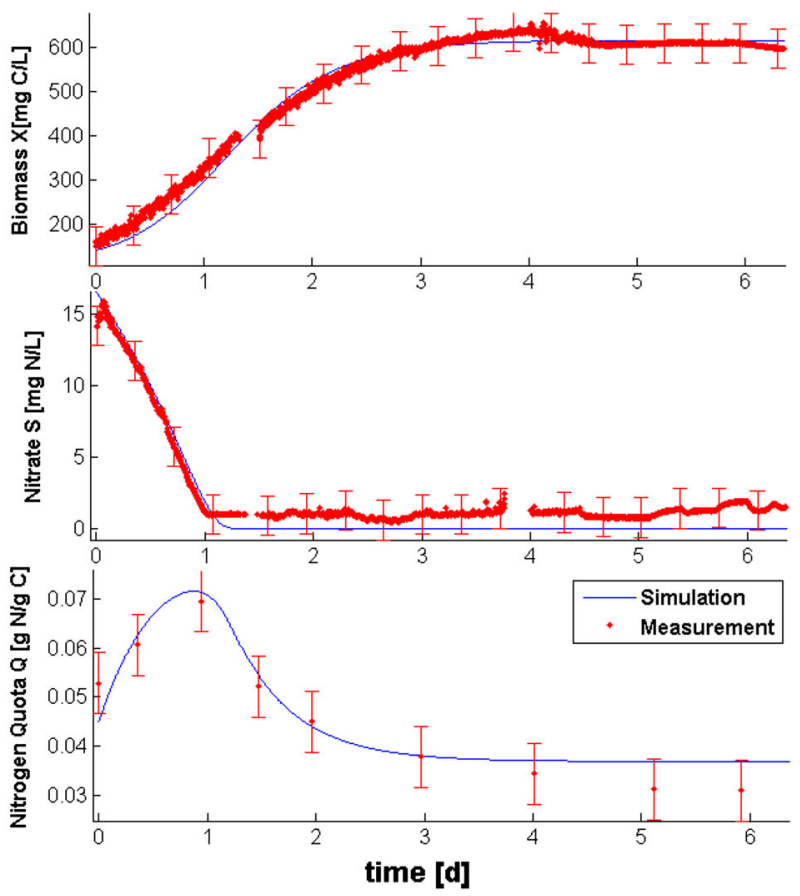

(b)

Fig. 7 Red dots experimental data, and blue lines model prediction—cross validation (color figure online) 
concentrations), and on the number of samples that can be collected during the experiments. Usually, a minimum of two experiments are considered, with different initial concentrations so as to highlight the influence of the several parameters.

Following this approach, two extra cultures (named third experiment and fourth experiment) are added to the dataset. The first two cultures will now be used for

Table 4 Initial conditions and coefficients of variation (CV) in cross validation

\begin{tabular}{llllll}
\hline Initial conditions & \multicolumn{2}{l}{ Third exp. } & & \multicolumn{2}{l}{ Fourth exp. } \\
\cline { 2 - 3 } & Value & CV & & Value & CV \\
\hline$X(0)$ & 135 & 4.49 & & 140 & 3.48 \\
$S(0)$ & 14.5 & 2.56 & & 16.5 & 1.79 \\
$Q(0)$ & 0.028 & 12.95 & & 0.045 & 6.79 \\
\hline
\end{tabular}

parameter estimation and direct validation, while the last two will be used for cross validation (i.e., to test the predictive capability of the model with fresh data). The results are presented in Table 3.

Figure 6 shows the direct validation results (red dots for the measurements, and blue lines for the model prediction). Apparently, the model fit is satisfactory.

Cross validation can then be used to evaluate how the model can reproduce the two next experiments, as shown in Fig. 7. Notice that for the last experiment, both the biomass concentration and the substrate concentration could be measured on-line.

The Initial condition values for these last two experiments have to be estimated and are listed in Table 4 together with their coefficients of variation.

Even though the model prediction is satisfactory in the first few days, it deteriorates on the long term when biomass is in a maintenance phase and it is observed that the
Table 5 Third parameter and initial condition estimates

\begin{tabular}{llllclcc}
\hline & Third estimates & CV $(\%)$ & \multicolumn{2}{l}{ Initial conditions } \\
\cline { 4 - 7 } & & & & First exp. & CV & Second exp. & CV \\
\hline$\rho_{\mathrm{m}}$ & 0.0817 & 1.48 & $X(0)$ & 125.02 & 0.59 & 224.405 & 0.44 \\
$K_{\mathrm{s}}$ & 1.9268 & 6.64 & $S(0)$ & 15.09 & 0.28 & 17.161 & 0.32 \\
$\mu_{\mathrm{m}}$ & 1.2088 & 0.66 & $Q(0)$ & 0.036 & 0.97 & 0.0351 & 0.91 \\
$Q_{0}$ & 0.0344 & 0.21 & & & & & \\
$\delta$ & 0.0522 & 0.19 & & & & & \\
\hline
\end{tabular}

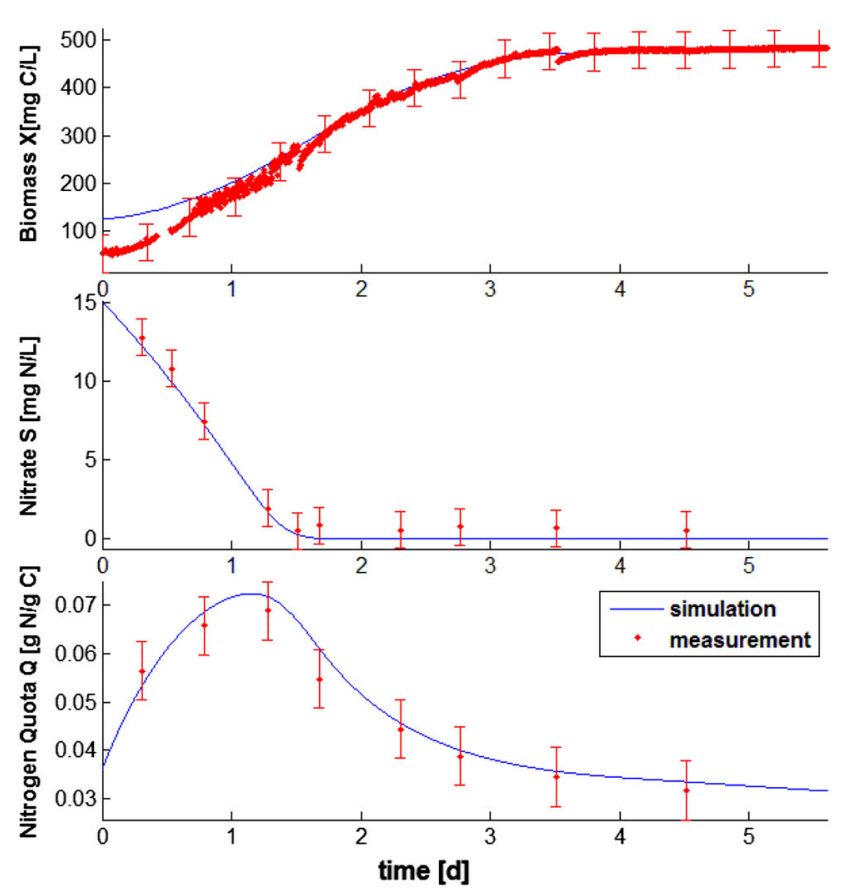

(a)
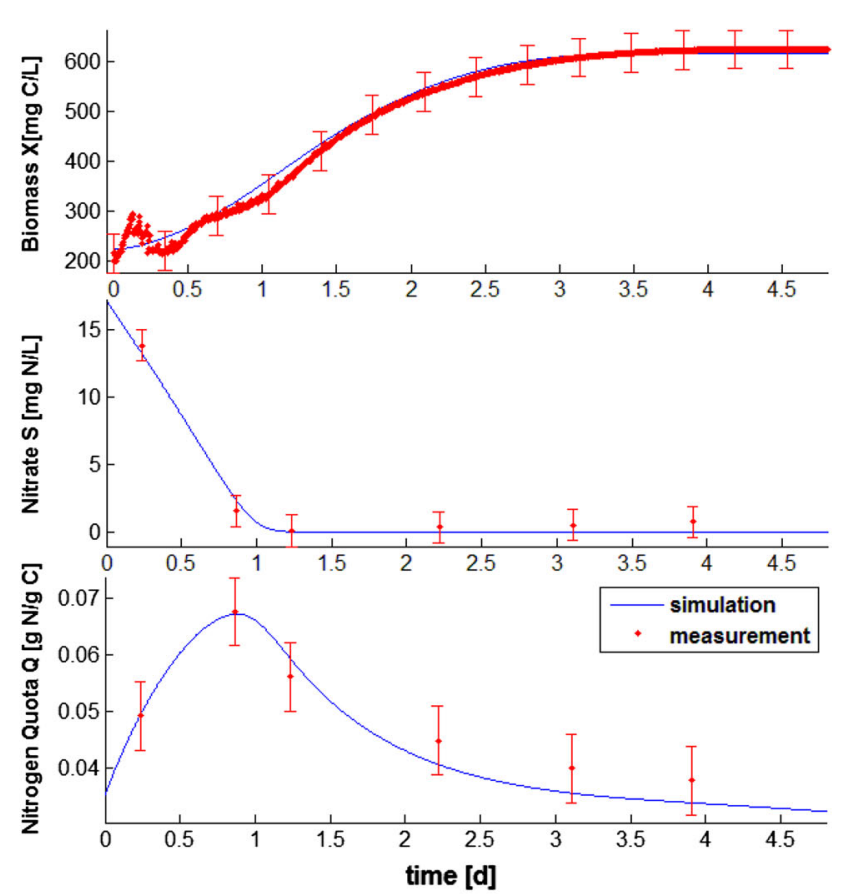

(b)

Fig. 8 Red dots experimental data, and blue lines model prediction-direct validation (color figure online) 


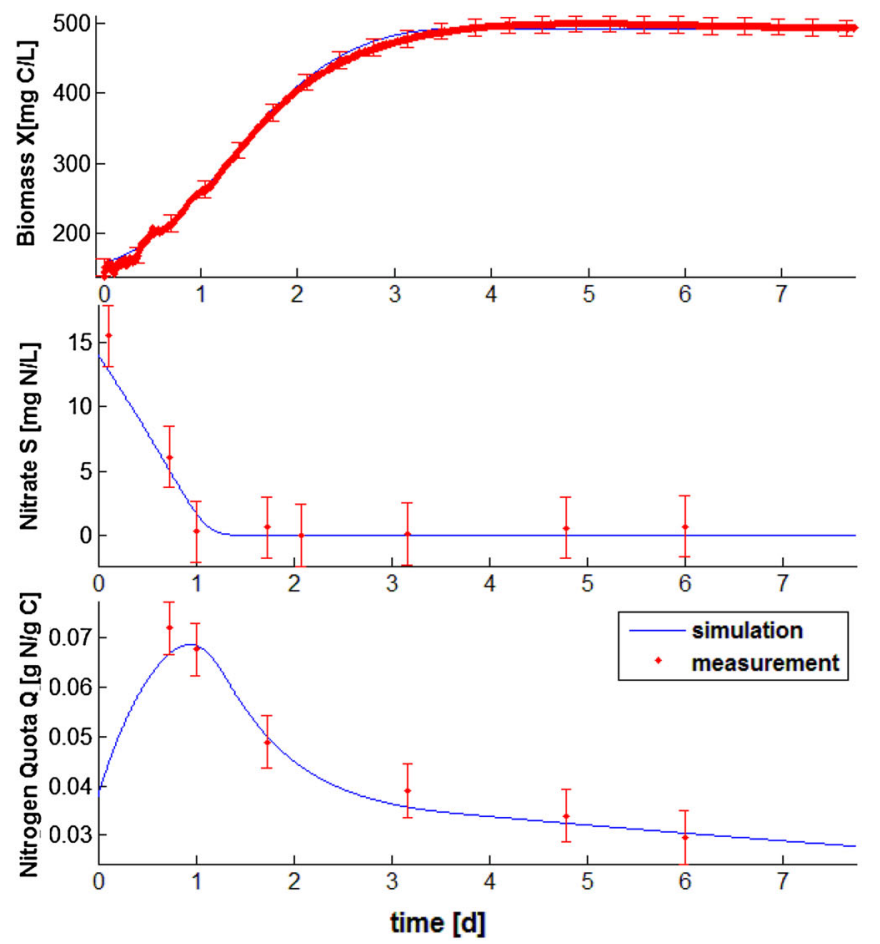

(a)

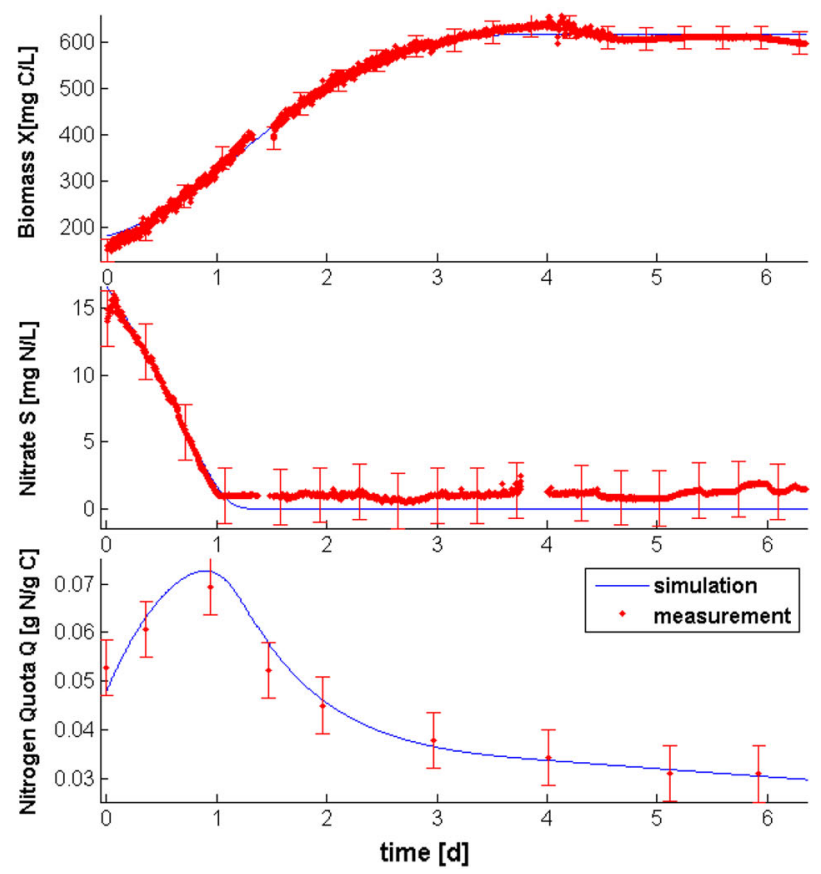

(b)

Fig. 9 Red dots experimental data, and blue lines model prediction-cross validation (color figure online)

Table 6 Initial conditions and coefficients of variation (CV) in cross validation

\begin{tabular}{llllll}
\hline \multirow{2}{*}{ Initial conditions } & \multicolumn{2}{l}{ Third exp. } & & \multicolumn{2}{l}{ Fourth exp. } \\
\cline { 2 - 3 } & Value & $\mathrm{CV}$ & & Value & $\mathrm{CV}$ \\
\hline$X(0)$ & 160 & 2.14 & & 180 & 1.63 \\
$S(0)$ & 14 & 2.08 & & 16.6 & 1.63 \\
$Q(0)$ & 0.038 & 6.46 & & 0.048 & 3.9 \\
\hline
\end{tabular}

internal quota decays. To reproduce the internal quota decay, it is proposed to introduce an additional term in the third equation of Eq. 4 with an unknown parameter $\delta$. Note that this situation is however not very interesting from a practical point of view as long-term culture would be achieved in continuous mode, where this phenomenon is not observed.

$$
\begin{aligned}
\dot{X} & =\mu(Q) X \\
\dot{S} & =-\rho(S) X \\
\dot{Q} & =\rho(S)-\mu(Q) Q-\delta Q
\end{aligned}
$$

A new parameter estimation is carried out considering the model of Eq. 18 and the new set of estimated parameters and initial conditions calculated are specified in Table 5. The direct validation of the new model is shown in Fig. 8, while cross validation is achieved with the two remaining sets of experimental data, and shown in Fig. 9 with the initial conditions given in Table 6. We can observe that the introduction of parameter $\delta$ allows a better reproduction of the final phase of the culture and the internal quota decay.

\section{Conclusions}

In this report, a lab-scale experimental set-up for the cultivation of microalgae in flat-panel photobioreactors is described, together with the associated on-line and off-line instrumentation. This set-up allows the collection of experimental data, and the development of dynamic mathematical models that could be used for process optimization and control. As a first application example, the parameters of a Droop model for the microalgae $D$. tertiolecta are estimated using nonlinear least-squares, from the experimental data collected from batch experiments. Direct and cross-validation results show the good model predictive capability. Future prospects include the implementation of continuous mode to collected data in various operating modes, and the study of additional environmental factors, including illumination. The developed platform is relatively low cost and allows easy further extensions. Among these, a novel RGB sensor for measuring biomass on-line has recently been proposed [16].

Acknowledgments This paper presents research results of the Belgian Network DYSCO (Dynamical Systems, Control, and 
Optimization), funded by the Interuniversity Attraction Poles Programme, initiated by the Belgian State, Science Policy Office (BELSPO). The authors are grateful to Olivier Bernard (INRIA-Sofia Antipolis) for providing the microalgal strains.

\section{References}

1. Qiang H, Sommerfeld M, Jarvis E, Ghirardi M, Posewitz M, Seibert M, Darzins A (2008) Microalgal triacylglycerols as feedstocks for biofuel production: perspectives and advances. Plant J 54(4):621-639

2. Chisti Y (2007) Biodiesel from microalgae. Biotechnol Adv 25(3):294-306

3. Becker EW (1993) Microalgae: biotechnology and microbiology, vol 10. Cambridge University Press, Cambridge

4. Bogaerts Ph, Vande Wouwer A (2003) Software sensors for bioprocesses. ISA Trans 42(4):547-558

5. Droop MR (1983) 25 years of algal growth kinetics a personal view. Bot Mar 26(3):99-112

6. Geider RJ, MacIntyre HL, Kana TM (1998) A dynamic regulatory model of phytoplanktonic acclimation to light, nutrients, and temperature. Limnol Oceanogr, 679-694

7. Mairet F, Bernard O, Masci P, Lacour T, Sciandra A (2011) Modelling neutral lipid production by the microalga Isochrysis aff. galbana under nitrogen limitation. Bioresour technol 102(1):142-149
8. Bernard O (2011) Hurdles and challenges for modelling and control of microalgae for $\mathrm{CO}_{2}$ mitigation and biofuel production. J Process Control

9. Droop MR (1968) Vitamin b12 and marine ecology. iv. the kinetics of uptake, growth and inhibition in monochrysis lutheri. J Mar Biol Assoc UK 48(3):689-733

10. Bernard O, Rémond B (2012) Validation of a simple model accounting for light and temperature effect on microalgal growth. Bioresour Technol

11. Bernard O, Gouzé J-L (1995) Transient behavior of biological loop models, with application to the Droop model. Math Biosci 127(1):19-43

12. Karlsson M, Karlberg B, Olsson RJO (1995) Determination of nitrate in municipal waste water by uv spectroscopy. Anal Chim Acta 312(1):107-113, 1995

13. Water Environment Federation American Public Health Association (1999) American Water Works Association. Standard methods for the examination of water and wastewater

14. Walter E, Pronzato L (1997) Identification of parametric models. Commun Cont Eng. Springer, New York

15. Dugdale RC (1967) Nutrient limitation in the sea: dynamics, identification, and significance. Limnol Oceanogr, 685-695

16. Benavides M, Mailier J, Hantson A-L, Muñoz G, Vargas A, Van Impe J, Vande Wouwer A (2015) Design and test of a low-cost rgb sensor for online measurement of microalgae concentration within a photo-bioreactor. Sensors 15:4766-4780 